\author{
Disponible on-line en: \\ http://revistalogopedia.uclm.es
}

UNIVERSIDAD DE

Revista de Investigación en Logopedia 2 (2014) 132-150.

ISSN-2174-5218

\title{
Aplicación de los índices de perturbación integrado y de precisión articulatoria en pacientes con disfonía espasmódica
}

\author{
Liliana Sigal, 1, 3 y Jorge A. Gurlekian, 2, 3 \\ 1, Hospital de Clínicas "José de San Martín”, Argentina. \\ 2, Laboratorio de Investigaciones Sensoriales-INIGEM CONICET, Argentina \\ 3 Universidad de Buenos Aires, Argentina.
}

\begin{abstract}
Resumen
La disfonía espasmódica es un desorden vocal severo caracterizado por interrupciones involuntarias de la fonación, denominada también distonía focal laríngea. Este trabajo da a conocer resultados de la aplicación del índice de perturbación integrado (IPI), y de precisión articulatoria (IPA), en una población de pacientes de ambos sexos con disfonía espasmódica, y presenta la comparación con un grupo control, sujetos con diagnóstico otorrinolaringológico de cuerdas vocales móviles, sin daños estructurales en la mucosa glótica, ni alteraciones neurológicas que afecten la dinámica cordal. El índice de perturbación agrupa a cuatro parámetros: Jitter, Shimmer, relación Armónico/Ruido y la Amplitud Diferencial del Cepstrum. Los resultados muestran diferencias significativas entre los grupos de casos y controles femeninos y masculinos en los parámetros de índice de perturbación integrado y de grado de precisión articulatoria, por lo que ambos pueden considerarse indicadores apropiados para caracterizar la falta de estabilidad en la función articulatoria que se produce en las cavidades resonanciales de las pacientes con disfonía espasmódica.
\end{abstract}

Palabras clave: Análisis acústico; Disfonía espasmódica; Índice de perturbación integrado.

\section{Application of integrated perturbation index and articulatory precision index in patients with spasmodic dysphonia}

\begin{abstract}
Spasmodic dysphonia -also called focal laryngeal dystonia- is a severe vocal disorder which presents nonvoluntary phonation breaks. This paper shows measurement results obtained by three indexes: 1) an integrated perturbation index (IPI), composed by four well known parameters: Jitter, Shimmer, Harmonic/Noise relation, and Cepstral peak amplitude and, 2) an articulatory precision index (IPA) for a population of patients with spasmodic dysphonia. These results are compared with a control set composed by patients with a laryngologist diagnose of mobile vocal folds with neither structural damages in the glotal mucosa, nor neurologic perturbations which could affect dynamics of the vocal folds. Results show clear differences between both groups for IPI and also for IPA. These indexes can be used as appropriated indicators to point out the lack of articulatory stability produced at the vocal resonators of patients with spasmodic dysphonia.
\end{abstract}

Key words: Acoustic analysis; Integrated perturbation index; Spasmodic Dysphonia.

Correspondencia con los autores: lilianasigal@yahoo.com.ar

Recibido 18 agosto. Primera revisión 9 octubre. Aceptado 18 de noviembre. 


\section{Introducción}

Traube (1871) fue el primer investigador en describir la disfonía espasmódica. La caracterizó como una ronquera de origen nervioso. Por muchos años fue llamada disfonía espástica, pero el término disfonía espasmódica es más aceptado en el mundo en estos días. Aronson, Brown, Litin y Pearson, (1968) fueron los investigadores que cambiaron el nombre de disfonía espástica por el de disfonía espasmódica (DE), argumentando que la espasticidad es una alteración específica de vías piramidales, y provoca rigidez, no espasmos como los observables en la laringe.

Pitman, Bliznikas y Baredes (2006) refirieron que la disfonía espasmódica es un desorden crónico de la voz, caracterizado por una excesiva o inapropiada contracción de los músculos laríngeos durante el habla.

Watts, Whurr y Nye (2007) escribieron que la disfonía espasmódica es un trastorno de la voz que es resultado de un control motriz laríngeo interrumpido que causa movimientos involuntarios de la musculatura laríngea durante la fonación. Estos movimientos involuntarios pueden causar que los pliegues vocales alcancen una hiperaducción inadecuada y se cierren (disfonía espasmódica aductora), o una abducción y se abran (disfonía espasmódica abductora), o en algunos casos ambas (Aronson y Bless, 1990; Cannito 1981). La disfonía espasmódica aductora es la forma más común del trastorno y se caracteriza por un esfuerzo muscular laríngeo, una calidad de voz tensaahogada y áspera, quiebres en la inflexión de la voz y frecuencia fundamental anormalmente baja (fricción de la glotis). La disfonía espasmódica abductora se caracteriza por el ensanchamiento intermitente de la glotis y una calidad de voz transitoriamente jadeante (Blitzer 1991; Aronson y Bless, 1990; Cannito 1981). La gravedad de los síntomas y la naturaleza incapacitante del trastorno puede variar de paciente a paciente. La comprensión de la etiología de la disfonía espasmódica ha evolucionado con el transcurso del tiempo, desde teorías de causas psicológicas subyacentes, hasta la opinión actual que enfatiza una causa neurológica primaria (Whurr, Lorch, Fontana, Brookes, Lees y Marsden, 1993; Cannito 2001). Como un tipo de distonía, la disfonía espasmódica se ha caracterizado como un trastorno neurológico crónico del procesamiento motriz central que causa espasmos musculares inducidos por la acción (Brin, Blitzer, Stewart, Diamond, y Pogoda, 2001).

Braden y Hapner (2008) explicaron que la disfonía espasmódica es una distonía focal de la laringe. Estos autores refieren que la disfonía espasmódica aductora compromete a los músculos laríngeos aductores y produce los síntomas de aspereza, 
sonidos parecidos al staccato y cortes en la fonación. La disfonía espasmódica abductora afecta a los músculos laríngeos abductores, resultando en una calidad vocal soplada o susurrada e interrupciones vocales. La disfonía espasmódica tiene un impacto significativo en la calidad de vida.

Kendall y Leonard (2009) también afirman que la disfonía espasmódica es una distonía laríngea crónica que afecta los músculos de la laringe, caracterizada por espasmos en las cuerdas vocales que resultan en una cualidad vocal tenso-estrangulada o con quiebres vocales suspirosos, dependientes de los músculos comprometidos. Refieren que el tipo aductor es el más común y que un $25 \%$ de estos pacientes también exhiben tremor vocal. El tipo abductor compromete al músculo cricoaritenoideo y el tipo mixto, que es menos común, a los dos: aductores y abductores. Los pacientes que presentan un significativo componente de tremor vocal al examen laríngeo revelan un desplazamiento rítmico medial de los cartílagos aritenoides. El movimiento compromete el cuerpo de los cartílagos aritenoides con la contracción de los músculos interaritenoideos.

Respecto a la patofisiología Simonyan, Ludlow y Vortmeyer (2010) definieron a la disfonía espasmódica como una distonía focal laríngea de patofisiología desconocida, caracterizada por espasmos involuntarios en los músculos laríngeos durante la producción de habla. En las distonías laríngeas hay alteraciones del tono muscular que afectan la movilidad de cuerdas vocales.

La American Speech-LanguageHearing Association (ASHA) escribe que en la disfonía espasmódica el movimiento de las cuerdas vocales es tenso y forzado y tiene como resultado una voz que suena quebrada, temblorosa, ronca, tensa o entrecortada. Se dan espasmos o interrupciones vocales, períodos durante los cuales no se produce sonido alguno y otros en los cuales aparece una voz casi normal.

\section{Estudios acústicos efectuados en pacientes con disfonías espasmódicas}

Ludlow, Naunton, Sedory, Schulz y Hallett, (1988), encontraron segmentos aperiódicos, cambios de frecuencia y cortes fonatorios en la emisión de frases. Sapienza y Walton (1999), por su parte, describieron estas mismas alteraciones durante la producción de vocales sostenidas. Edgar, Sapienza, Bidus, Ludlow (2001) propusieron el estudio acústico con la producción del máximo tiempo fonatorio con la vocal /a/ y la repetición de 4 oraciones. Investigaron la duración de la palabra y de la oración, del inicio fonatorio, el número de cambios frecuenciales, de segmentos aperiódicos y de interrupciones fonatorias y la duración de las interrupciones fonatorias. 
Cannito, Buder y Chorna (2005) demostraron la aplicabilidad clínica de la medición de las diferencias de amplitud de los armónicos en el habla para caracterizar la fonación de personas con disfonía espasmódica, comparándolas con un grupo control de hablantes normales. Sugirieron que las medidas de la amplitud espectral son sensibles a variados grados de aducción cordal.

Roy, Whitchurch, Merrill, Houtz y Smith (2008), realizaron un estudio sobre el análisis de quiebres fonatorios durante la emisión de una frase. Investigaron a dos grupos de pacientes: con disfonías espasmódicas aductoras y con disfonías por tensión muscular. Estos autores determinaron la presencia, frecuencia y duración de los quiebres fonatorios, definidos como una completa interrupción de la fonación. Encontraron que el grupo de pacientes con DE presentó un número más alto de quiebres comparados a las disfonías por tensión muscular. Este estudio ha sido considerado como un posible test objetivo con precisión diagnóstica para distinguir ambas patologías vocales.

Siemons, Moerman, Marten, Deuster, Müller y Dejonckere (2009) han estudiado mediante el sistema AMPEX el porcentaje de fragmentos de voz, la duración y promedio de lo sonoro, el valor del índice Jitter y otros parámetros durante la emisión de vocales, sílabas y frases. Aplicaron una escala de calificación perceptual: la IINFVo: cuyas siglas significan impresión, inteligibilidad, ruido, fluidez y sonoridad. Se demostraron correlaciones altamente significativas entre la escala perceptual y la acústica, excepto en el parámetro de ruido, considerándose a AMPEX e IINFVo herramientas de evaluación robustas y complementarias para la evaluación de la disfonía espasmódica de aducción. Éstas proporcionan información valiosa acerca de la calidad de voz, la estabilidad de F0 (frecuencia fundamental) y las dimensiones específicas que controlan las transiciones entre los segmentos sonoros y sordos. Los autores proponen, para otros trabajos futuros, el análisis cepstral aplicado al habla conectada, como método más eficiente para el estudio de la aperiodicidad en las disfonías espasmódicas de aducción. Asimismo las diferencias en la amplitud de los armónicos, cuyos resultados pueden ser una estrategia viable para la cuantificación.

Watts et al., (2007), proponen estudios de la voz y de la función respiratoria, previos a las intervenciones experimentales con inyección unilateral o bilateral de toxina botulínica en uno o más músculos del mecanismo laríngeo, denominando a estos estudios: tipos de medidas de resultado, y las describen: 1) La función acústica, o la medición instrumental del espectro de la voz mediante un análisis computadorizado (p.ej. frecuencia fundamental, perturbación de la frecuencia y de la amplitud, cociente señal/ruido). 2) Las calificaciones 
perceptuales, o las calificaciones subjetivas de la calidad de la voz o la capacidad (p.ej. mejoría subjetiva de la voz, calificaciones espectrográficas de normalidad). 3) La función aerodinámica, o la medición instrumental del flujo respiratorio y la presión de aire que se necesita para hacer vibrar los pliegues vocales (p.ej. flujo aéreo medio, coeficiente de variación de flujo aéreo, presión del aire).

Whitchurch (2008) le da valor diagnóstico al análisis acústico de los quiebres fonatorios como un posible test objetivo para discriminar la disfonía espasmódica aductora de las disfonías por tensión muscular. Grabó a 41 pacientes con cada una de las citadas alteraciones, la lectura de una oración confeccionada con fonemas sonoros. Midió la presencia y duración de cada interrupción fonatoria dentro de las palabras emitidas. Concluyó que si un paciente tiene más de 4 interrupciones se considera que tiene una disfonía espasmódica. Además citó la definición de Sapienza y Walton (1999) consistente en definir el quiebre fonatorio como una interrupción en sonoridad de la palabra mayor a 50 ms. Whitchurch (2008) consideró también como quiebres fonatorios a las interrupciones mayores a $20 \mathrm{~ms}$. para establecer el diagnóstico diferencial entre disfonía espasmódica aductora y por tensión muscular. La duración absoluta del break fonatorio fue medida en milisegundos, marcando el inicio del quiebre en el último y claro pulso glotal positivo, y el final del quiebre en el retorno de la fonación. Refirió que se podía encontrar más de un corte en cada palabra. Por último presentó un ejemplo de una interrupción en la palabra “mile” cuya duración fue de 100.93 ms. Investigó la presencia, número y duración de los quiebres vocales, diferencias entre hombres y mujeres, y el valor del análisis de los breaks o interrupciones como herramienta en el diagnóstico diferencial.

Kendall y Leonard (2009) han tratado pacientes con disfonía espasmódica y tremor con toxina botulínica. Estos pacientes fueron grabados pre y post tratamiento. Las muestras incluyeron cinco repeticiones de /i/ ("ee") producida en una frecuencia confortable, con duración de 5 a 7 segundos. En las vocales se seleccionaron fragmentos de 1.5 a 2 segundos, a partir de la zona media de la emisión, para analizar estas perturbaciones de Frecuencia Fundamental: Jitter (\%) y Shimmer (\%). Para el análisis de Rango de Frecuencia Fundamental (evaluada en semitonos) y la desviación estándar (en Hz.) se utilizaron la duración total de vocales.

Cannito, Buder, Chorna y Dressler (2012) opinan que la mayoría de los estudios se han focalizado al análisis acústico de la emisión vocálica. No obstante la fonación en habla conectada es el criterio más actual para juzgar la comunicación funcional del paciente con disfonía espasmódica aductora, y para evaluar la respuesta al tratamiento. Estos autores 
realizaron un estudio midiendo eventos acústicos en frecuencias bajas, el coeficiente de variación de F0 en registro modal, y la relación señal-ruido. Examinaron las medidas acústicas en la producción de segmentos de habla de los pacientes con disfonía espasmódica aductora antes y después de la toxina botulínica, y las compararon con el habla de un grupo control de sujetos sanos sin disfonía. Realizaron correlaciones entre las medidas acústicas y perceptivas. Se emplearon algoritmos de procesamiento de señal para extraer la frecuencia fundamental (F0) y la intensidad (dB) Se midió el número de eventos de baja frecuencia, el coeficiente de Variación de la F0 modal y el índice armónicos/ruido. La media de F0 en registro modal no se diferenció entre pacientes y sujetos control. El índice SNR demostró diferencias estadísticamente significativas en el habla de los pacientes con ADSD antes y después del tratamiento y diferenció a los pacientes con ADSD y los normales. Los autores también destacan la futura utilización del análisis cepstral aplicado al habla conectada y las diferencias en la amplitud de los armónicos.

Cannito, Doiuchi, Murry y Woodson (2012) realizaron un trabajo de relación entre resultados perceptuales y acústicos de una población de pacientes con disfonía espasmódica aductora, previo y post la inyección de toxina botulínica. De una lectura fonéticamente balanceada se extrajeron 15 palabras sonoras. Se investigó el porcentaje de quiebres fonatorios, de segmentos aperiódicos, de cambios frecuenciales y el índice CPPs que es una modificación del Cepstrum. Este último es la única medida acústica con un valor predictivo para estimar soplosidad y tiene un fuerte correlato acústico de aspereza.

Dejonckere, Neumann, Moerman, Martens, Giordano y Manfredi, (2012), llevaron a cabo una investigación grabando 40 frases cortas fonéticamente balanceadas para investigar la sonoridad constante. Se evaluaron los pacientes mediante el examen perceptual y el acústico. En este último se estudiaron: 1- proporción de fragmentos sonoros: (PVF). 2-Promedio de evidencia sonora: grado de regularidad/periodicidad en los fragmentos sonoros ( AVE) . 3- El tradicional Jitter: Jit y el Jitc o Jitter corregido. Y el cuarto parámetro es el percentile 90 (VL 90) de la distribución sonora, que estima la duración máxima sonora.

Sigal (2013), siguiendo trabajos de Sapienza y Walton (1999), Edgar et al., (2001), Roy et al., (2008) y Whitchurch (2008), ha analizado acústicamente la vocal /a/ investigando el valor de la frecuencia fundamental, desvío estándar, rango diferencial entre frecuencia máxima y mínima y número de quiebres fonatorios, y ha comparado los resultados entre una población de pacientes con disfonía espasmódica y un grupo control. En los resultados de Sigal (2013) no se encontraron diferencias significativas entre las medias de F0 entre ambos 
grupos estudiados. La comparación de los promedios del desvío estándar de la Frecuencia Fundamental ha dado diferencias significativas, así como la comparación de los promedios de los rangos entre frecuencia máxima y mínima. Respecto a la variable quiebres fonatorios se calcularon en el grupo de pacientes. Ninguno de los sujetos del grupo control presentó interrupciones fonatorias en la emisión de la vocal. Como conclusión se expresa que la desviación estándar, el rango entre el valor máximo y mínimo de F0 y el número de interrupciones durante la emisión de vocal prolongada, pueden utilizarse como indicadores de disfonía espasmódica.

Hartmann, Hartmann, Heftner y Angerstein (2013) aplicaron a sus pacientes una lectura llamada “The northwind and the sun". Extrajeron las secuencias sin sonoridad entre palabras para investigar el habla sonora. Se compararon previo y posteriormente al tratamiento con toxina botulínica: la duración total de los pasajes sonoros, el Jitter y el Shimmer local y el número de quiebres o interrupciones sonoras.

\section{Estudio}

La mayoría de los trabajos ahora citados sobre disfonías espasmódicas se refiere a las alteraciones fonatorias, no encontrándose en la literatura evidencias directas a la contribución de los fenómenos articulatorios en las cavidades resonanciales. Cannito et al., (2012) mencionan en su trabajo la conveniencia de la utilización en el futuro de los componentes espectrales. En este trabajo se investigarán dichos fenómenos mediante dos índices: el índice de perturbación integrado (IPI), y de precisión articulatoria (IPA).

El IPA emplea información de la estabilidad de los formantes. Calcula la desviación estándar en la medición de los primeros cinco formantes. Esta medición permite cuantificar la falla que afecta al sostén de la posición articulatoria que se supone ocurre en las disfonías espasmódicas por inestabilidad fonatoria dada la presencia de espasmos y en algunos casos características asociadas al temblor o tremor vocal (Edgar, Barkmeier, Case, y Ludlow, 2001).

El IPI agrupa el Jitter, Shimmer, H/N y la Amplitud del Cepstrum. En virtud de la incertidumbre en la medición del Jitter utilizamos el Jitter \% a partir de una verificación de su confiabilidad en la medición de la frecuencia fundamental ciclo a ciclo (F0cc) que consiste en comparar el valor de F0cc con el valor de F0 promedio calculado mediante un método de mayor confiabilidad: función de correlación cruzada y programación dinámica GetF0, (Talkin, 1995). Como derivación del cálculo del Cepstrum se obtiene un parámetro frecuentemente utilizado en la literatura reciente: la amplitud diferencial del pico máximo 
del Cepstrum. Es decir la diferencia de amplitud del pico máximo, relativa a la amplitud interpolada de los parciales restantes y el ruido presente en la emisión.

Los dos índices mencionados se presentaron originalmente en un trabajo referido a la determinación del riesgo vocal en adultos (Gurlekian y Molina, 2012)

En este trabajo empleamos los índices mencionados IPI e IPA, que evalúan tanto la alteración de los componentes fonatorios como articulatorios, para aplicarlos en las disfonías espasmódicas.

\section{Método}

\section{Participantes}

El diseño de investigación es retrospectivo, transversal, descriptivo y comparativo. La población en estudio consiste en 83 pacientes con disfonía espasmódica de sexo femenino de 30 a 88 años (Media: 63,73; DE: 9,88) y un grupo control compuesto por 43 mujeres de 30 a 82 años (Media: 61,04; DE: 11,08). Se evaluaron 24 pacientes de sexo masculino de 30 a 82 años (Media: 60,75; DE: 14,37) con disfonía espasmódica y un grupo control de 24 sujetos de 30 a 84 años (Media: 53,83; DE: 15,53).

El grupo control se ha conformado por sujetos sin alteraciones en la estructura y dinámica cordal, cuyas voces se han evaluado perceptualmente mediante la escala perceptual GRBAS (Hirano, 1981). Se han incluido en este estudio a los sujetos cuyos resultados para el grado $\mathrm{G}$ son normal (0) y leve (1).

\section{Procedimiento}

El estudio acústico se realiza con la vocal /a/ sostenida, mediante una grabación de 3 segundos de duración, con micrófono unidireccional Ultravoice en ambiente silente (se seleccionó una de tres muestras de la vocal /a/ emitidas en tonalidad e intensidad confortables). Se emplearon las técnicas de procesamiento digital de la señal de habla muestreadas a $16 \mathrm{KHz}$ y 16 bits y analizadas una ventana de $16 \mathrm{~ms}$. y 6,25 ms. de desplazamiento. La señal se analiza en el segmento sonoro estable de mayor duración sin quiebres fonatorios. Los segmentos utilizados tienen una duración mínimo de 100 ms. y pueden alcanzar los 3 segundos.

Para el estudio acústico de ambas poblaciones: pacientes con disfonías espasmódicas y grupo control se emplea el sistema Anagraf (Gurlekian, 1997) que calcula los índices que se describen a continuación: 
Índice de Perturbación Integrado (IPI): agrupa 4 parámetros:

Jitter porcentual (\%): promedio de las diferencias de la frecuencia fundamental ciclo a ciclo, normalizadas al promedio de la F0 y multiplicada por 100.

Shimmer $(d B)$ : promedio de las diferencias de amplitud ciclo a ciclo convertidas a decibelios.

Relación Armónico-Ruido $(H / N)(d B)$ : diferencia entre la intensidad armónica y la intensidad de ruido.

Amplitud del Pico de Cepstrum (ACep) (dB): Diferencia de amplitud entre el pico de mayor amplitud en la zona de bajas frecuencias y la amplitud interpolada del resto. El Cepstrum se calcula como la transformada inversa de Fourier del logaritmo del espectro estimado de la señal.

\section{Gráficos del Índice de Perturbación Integrado:}

En la figura 1 se observa cómo se efectúa el cálculo de este índice mediante un gráfico que resume los rangos y contribuciones de cada parámetro. El cuadrado grande se halla subdividido en dos cuadrados más pequeños: uno abajo a la derecha (las escalas comprenden los valores de normalidad de los parámetros) y siguiendo la diagonal otro cuadrado en la zona de arriba a la izquierda (donde las escalas comprenden los valores alterados de los parámetros). Las escalas son lineales para los valores de H/N, Amplitud del Cepstrum y Shimmer y logarítmica para los valores del Jitter. El cuadrado de abajo a la derecha se subdivide a su vez en los segmentos de contribución $0,1,2$ (normalidad). El de arriba a la izquierda en las contribuciones 3, 4, 5 (alterados). El promedio de las cuatro contribuciones de los parámetros determina el índice IPI.

\section{Índice de Precisión Vocal Articulatoria (IPA)}

Se calcula a partir de las desviaciones estándar de los valores de frecuencia de los cinco formantes. La precisión se asocia con el mantenimiento de los valores de frecuencia de formantes a lo largo de la emisión. Un mínimo grado de imprecisión produce un efecto acústico similar al efecto positivo del vibrato y permite un ajuste por realimentación para obtener mayor energía a la salida. Cuando la imprecisión es mayor y no controlada el efecto desaparece y se corresponde con una alteración. En la Figura 2 se observa a la derecha el movimiento de los formantes 1 y 2 debido a la falta de sostén estable de la posición articulatoria. También hay un descenso del formante 1 debida a la nasalización. Los puntos que se observan en la elipse de la vocal/u/ corresponden al primer formante nasal. 
Los valores de normalidad hallados previamente por Gurlekian y Molina (2012) son para IPI menor a 2 y para IPA mayor a $90 \%$. A su vez se ha definido para IPI el rango de riesgo entre 2 y 3 y el de alteración mayor a 3.
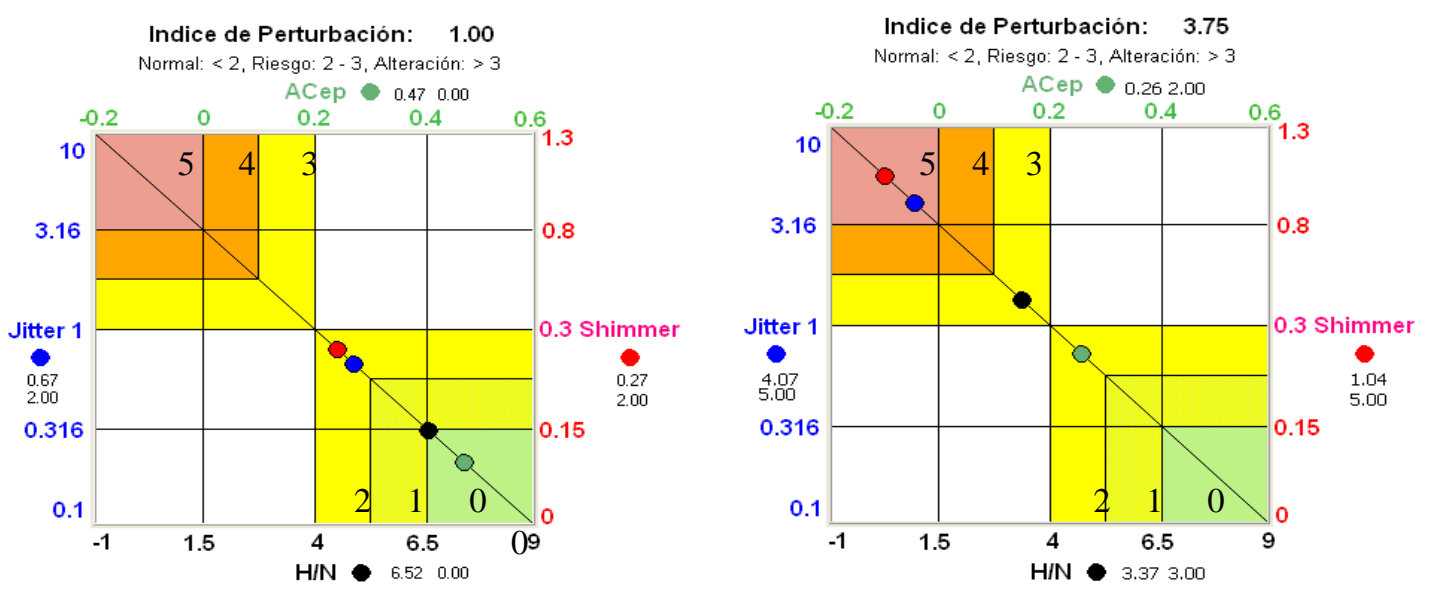

Figura 1. Dos ejemplos de IPI calculados durante la emisión de /a/ sostenida. A la izquierda: sujeto del grupo control que presenta los cuatro parámetros con contribuciones: $0,0,2$ y 2 (dentro de la normalidad) A la derecha un paciente con disfonía espasmódica con contribuciones: 2, 3, 5 y 5 (valores alterados). Los resultados de los índices de perturbación han dado: 1 y 3.75 respectivamente para el sujeto control y con disfonía espasmódica. (normal < a 2.00)
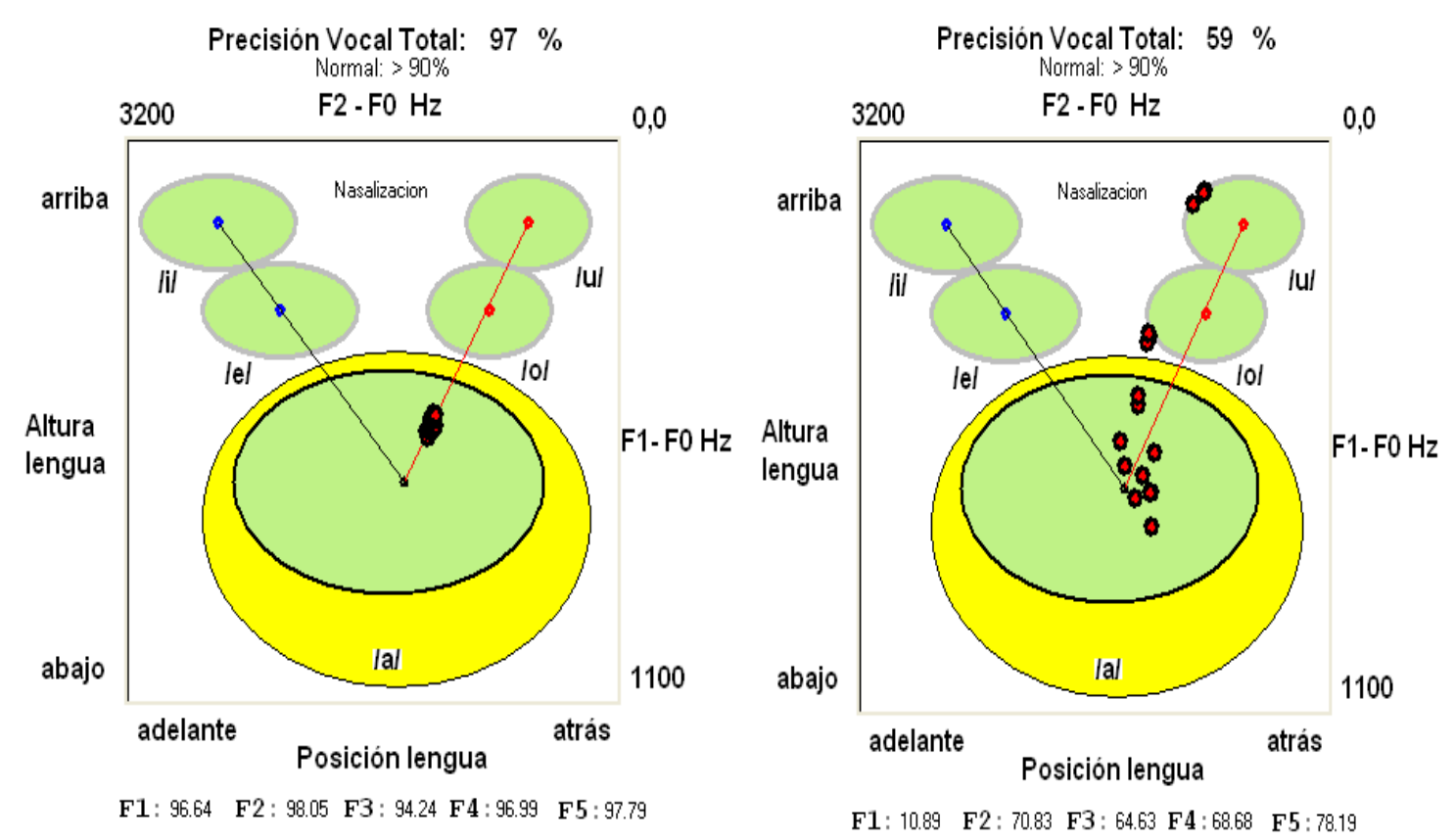

Figura 2. Dos ejemplos del Índice de Precisión Articulatoria (IPA) para la emisión de una /a/ sostenida. A la izquierda el resultado de un sujeto del grupo control: 97\%, a la derecha el resultado de un paciente con disfonía espasmódica: 59\%: disminuido. (Valor normal > a $90 \%$ ) Se grafica en el plano F1 vs F2, normalizado por F0. En la parte inferior de los gráficos se indican los valores promedio de precisión de cada uno de los cinco formantes. 
En la Figura 3 se presenta un perfil acústico de vocal /a/ sostenida emitida por una paciente de sexo femenino con disfonía espasmódica y en la Figura 4 se muestra el cálculo de los índices obtenidos del segmento seleccionado para una paciente.

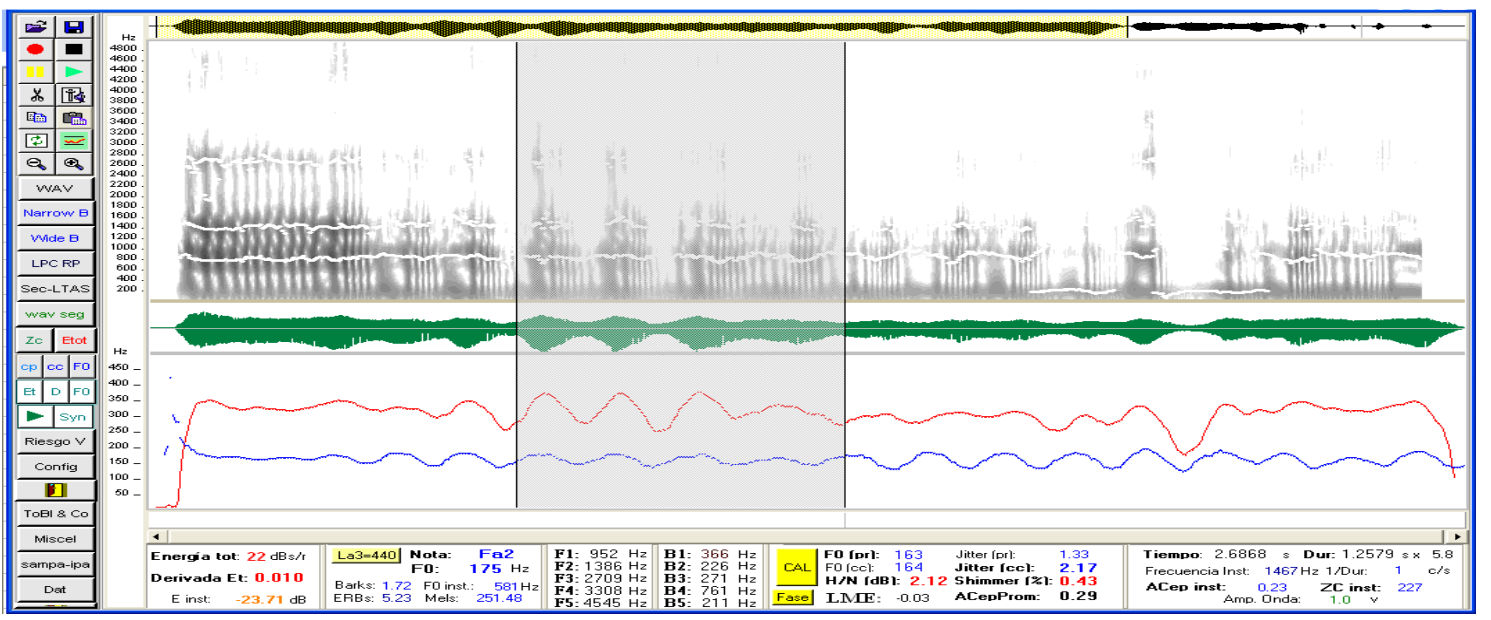

Figura 3. En ventana superior: espectro de banda ancha: inestabilidad en configuración de formantes. En el centro: forma de onda: oscilaciones. En ventana inferior el contorno de frecuencia: con cambios frecuenciales y amplitud: oscilaciones.

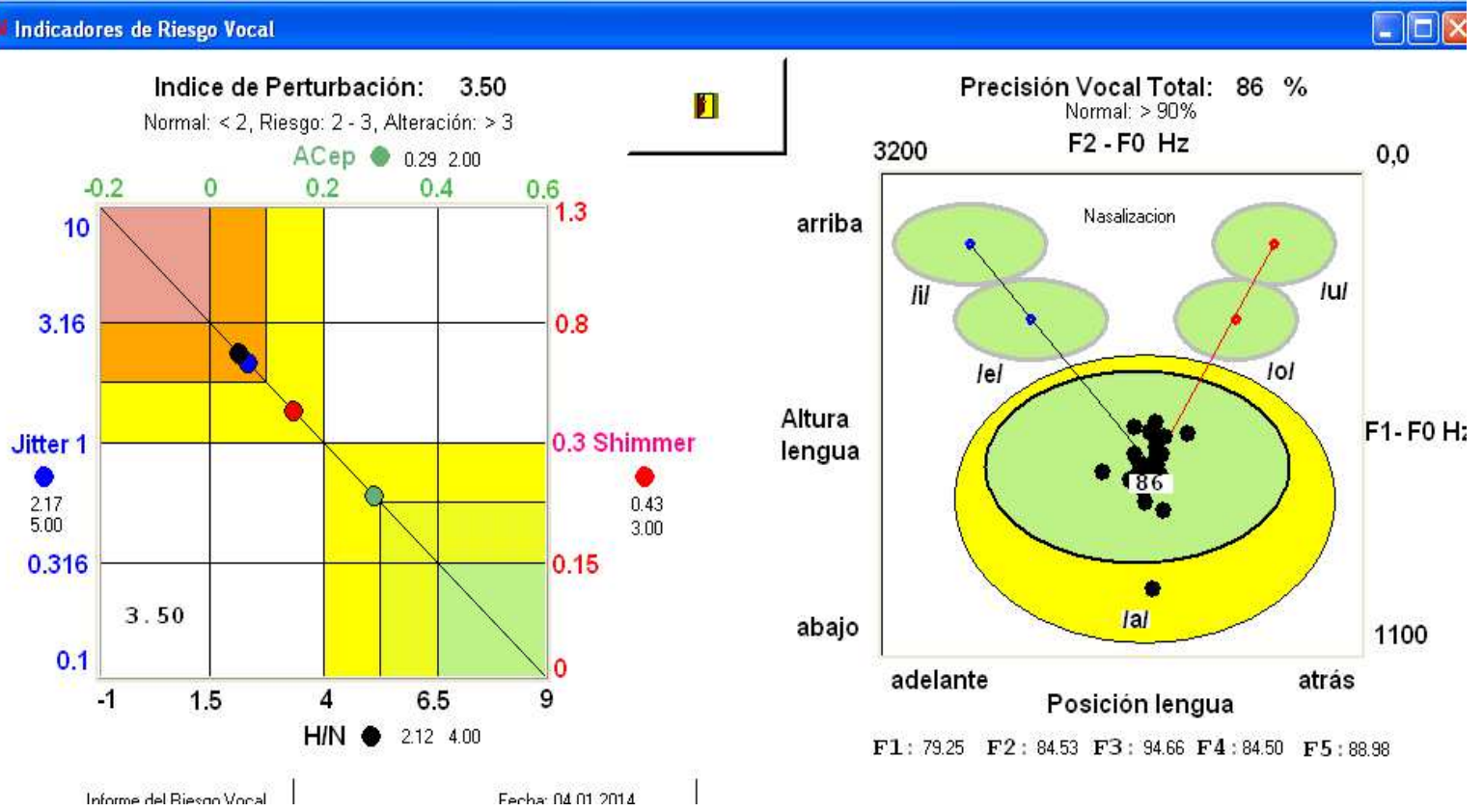

Figura 4. Representación gráfica del cálculo de los índices obtenidos del segmento seleccionado de /a/, de la citada paciente. Índice de perturbación integrado: IPI: 3.50: valor elevado (normal < a 2). Índice de Precisión Vocal Articulatoria: IPA: $86 \%$ : descendido (normal > a $90 \%$ ) 
El cálculo de los parámetros y de los índices se lleva a cabo en la población de pacientes con disfonía espasmódica, y en los sujetos del grupo control que presentan voz normal o con disfonías leves. Como se mencionó se seleccionó un segmento de la señal que no presentara quiebres ni interrupciones en su sonoridad, es decir con F0 continuo.

\section{Resultados}

Los resultados obtenidos en el grafico para la disfonía espasmódica muestran valores para las medianas del índice IPI, que llegan a duplicar a los del grupo control. Las diferencias en los valores del índice IPI entre los sujetos del grupo control con grado $G=0$ y 1 de acuerdo a GRBAS y con disfonía espasmódica son claramente significativas como lo indica el análisis de las varianzas ANOVA para mujeres $(\mathrm{F}=112.902, \mathrm{p}<.001)$ y para hombres $(\mathrm{F}=52.54, \mathrm{p}<.001)$.
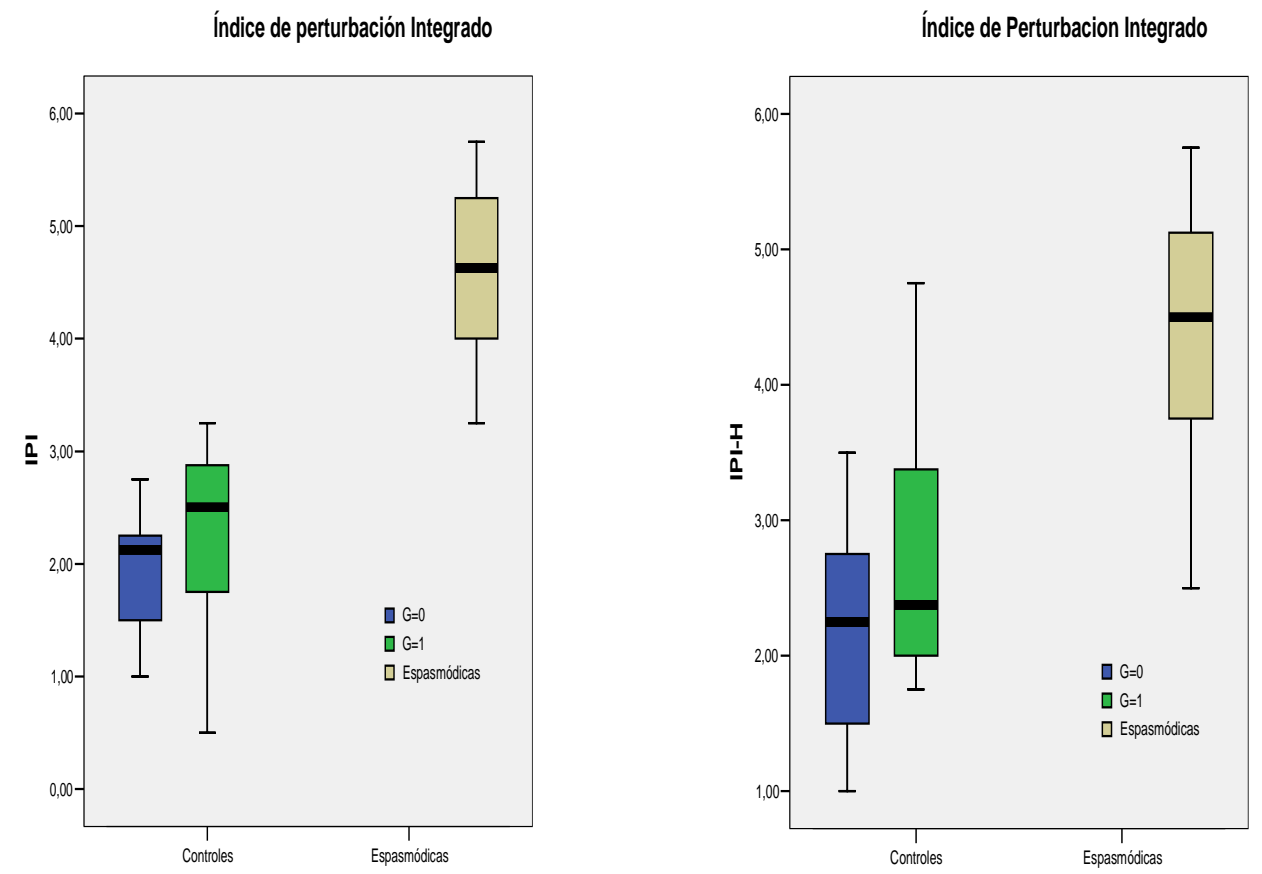

Figura 5. Gráficos de caja del índice IPI para Mujeres (izquierda) y Hombres (derecha). En cada grafico la población de controles está dividida en dos grupos según los resultados obtenidos por la escala GRBAS. (G= 0 y 1). A la derecha los pacientes con Disfonía Espasmódica. 

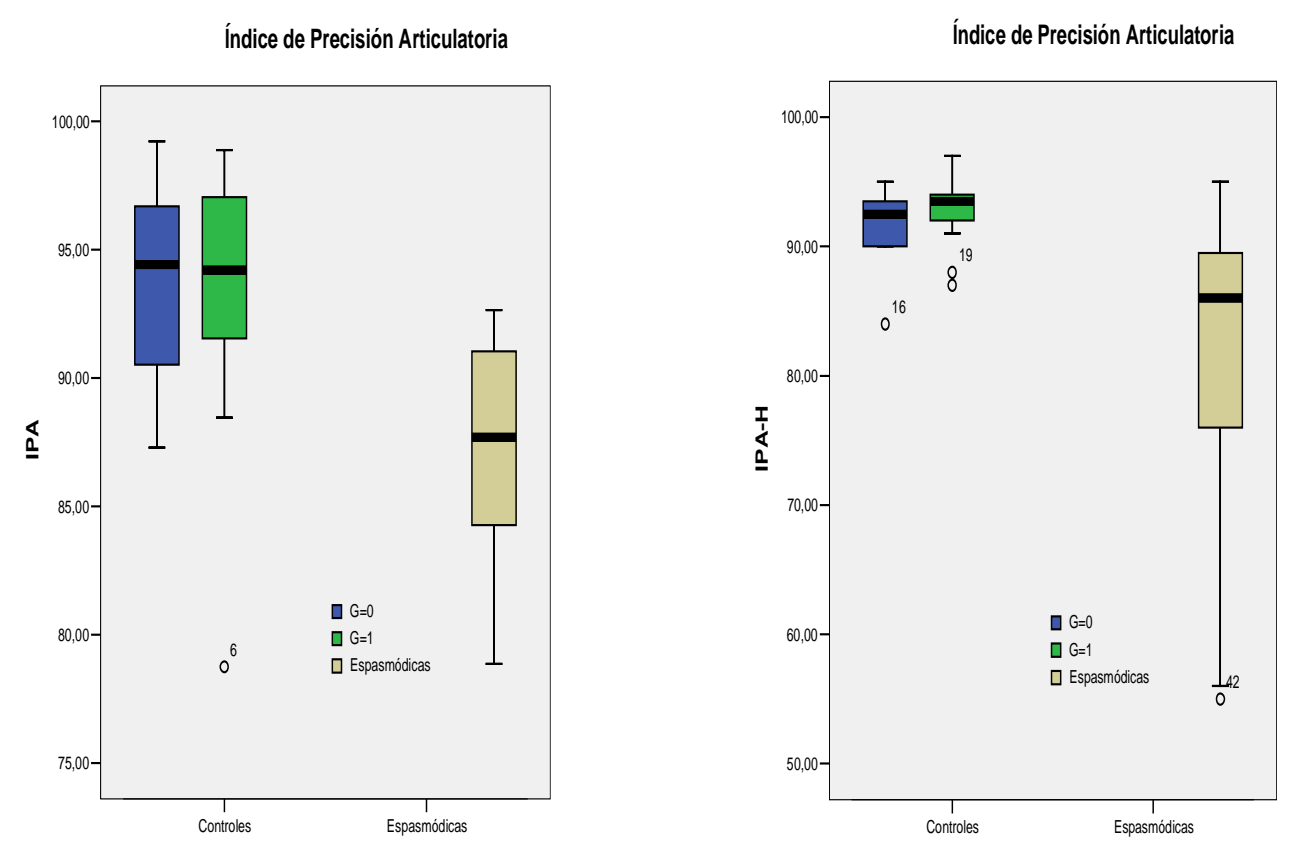

Figura 6. Gráficos de caja del índice IPA para Mujeres (izquierda) y Hombres (derecha). En cada grafico la población de controles está dividida en dos grupos según los resultados obtenidos por la escala GRBAS. (G= 0 y 1). A la derecha los pacientes con Disfonía Espasmódica.

Las diferencias en los valores del índice IPA entre los sujetos del grupo control con grado $\mathrm{G}=0$ y 1 de acuerdo a GRBAS y con disfonía espasmódica son significativas como lo indica el análisis de las varianzas ANOVA para mujeres $(\mathrm{F}=20.495, \mathrm{p}<.001)$ y para hombres $(\mathrm{F}=28.243, \mathrm{p}<.001)$.

El tamaño de la muestra permitió dividir los rangos etarios en grupos en función de una distribución balanceada de pacientes. Como se mencionó se seleccionó un segmento de la señal que no presentara quiebres ni interrupciones en su sonoridad, es decir con F0 continuo. Las figuras 7 y 8 presentan los resultados obtenidos para los dos índices estudiados. 


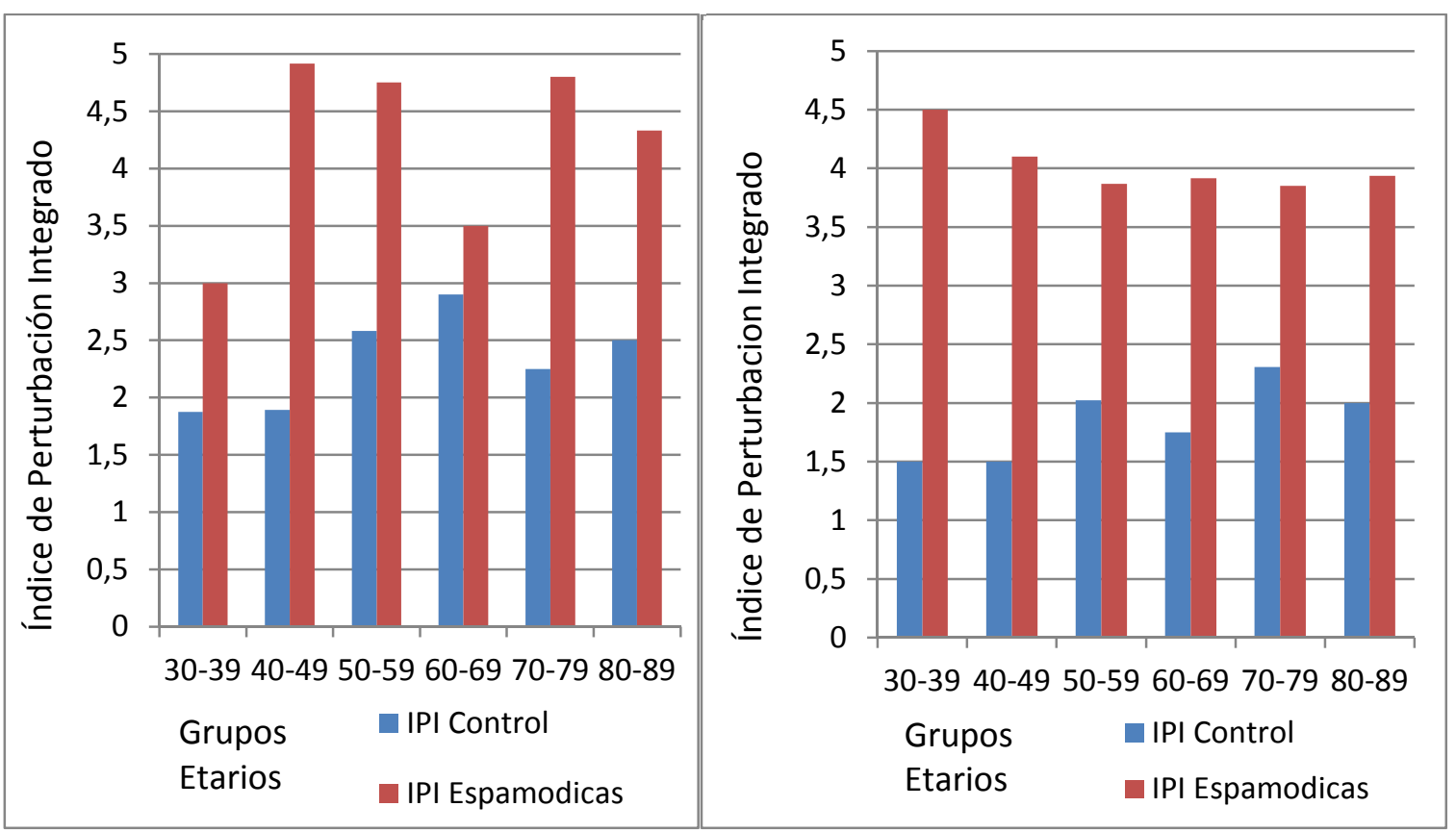

Figura 7. Valores promedio del índice de perturbación integrado (IPI) para cada rango etario en la población femenina (izquierda) y masculina (derecha). (Normal < 2.5).

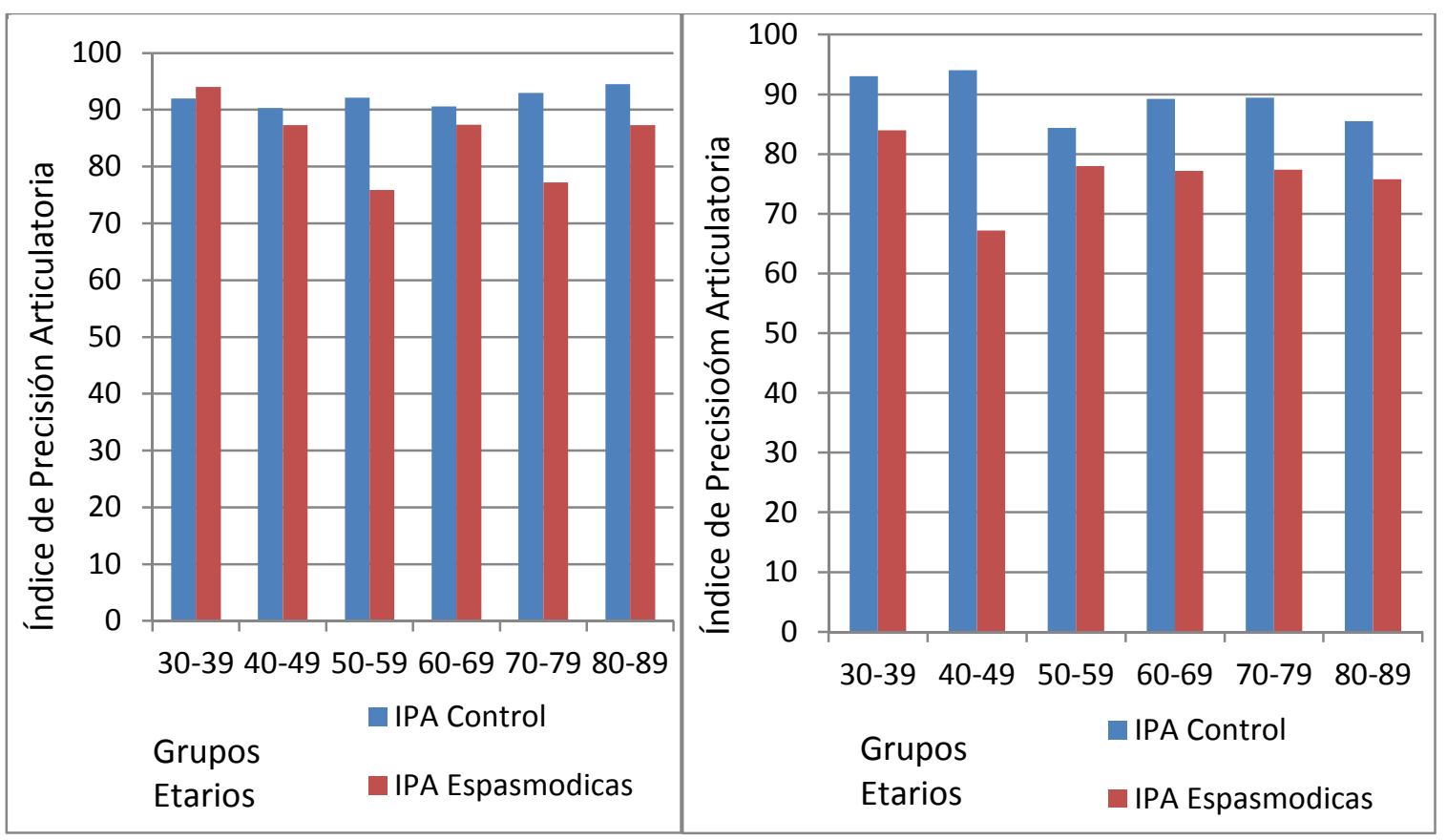

Figura 8. Valores promedio del índice de perturbación articulatoria (IPA) para cada rango etario en la población femenina (izquierda) y masculina (derecha). (Normal >90\%).

\section{Discusión}

La medición del índice de perturbación integra, mediante un cálculo gráfico automático (ver Figura 1), distintos parámetros que cuantifican tanto el grado de alteración fonatoria mediante el Jitter y Shimmer, como del aporte fonatorio-articulatorio del $\mathrm{H} / \mathrm{N}$ y la 
Amplitud del Cepstrum. Aun cuando los parámetros poseen distintas unidades, el método empleado permite obtener la contribución cada uno de ellos a la perturbación general con un número sin dimensión que permite calcular el promedio de las contribuciones parciales. El cálculo gráfico automático presenta distintas escalas que emulan el comportamiento psicoacústico de cada parámetro, en los rangos posibles de normalidad y alteración. El valor de cada parámetro acústico medido se proyecta a la diagonal que ha sido dividida en segmentos que representan los diferentes grados de perturbación.

Como se muestra en el ejemplo de la Figura 3, la medición se realiza en segmentos donde hay continuidad en los contornos de F0 y energía total para tener un criterio de medición similar, dado que algunos pacientes con disfonía espasmódica presentan interrupciones y otros no.

La aplicación del IPI ha mostrado ser claramente diferenciadora de las voces de los sujetos del grupo control y con disfonía espasmódica, dado que su diseño posee la sensibilidad adecuada a la detección de pacientes con riesgo vocal. Ver figuras 5 y 7 . Las categorías de pacientes normales $(<2)$, en riesgo (2-3) y con alteración (>3) permiten incluir claramente a las disfonías espasmódicas estudiadas en el grupo con alteración.

Como se mencionó en la introducción, los pacientes con disfonía espasmódica presentan particularmente alteraciones en el sostén de la posición articulatoria durante la producción de la vocal, por lo que resulta de interés analizar el comportamiento del Índice de Precisión Vocal Articulatoria -ver figura 2-. La modificación de los articuladores en las cavidades resonantes modifican los valores de formantes. La falta de estabilidad en la producción de los formantes es cuantificada a partir de la desviación estándar, cuya inversa ha sido denominada precisión vocal articulatoria. Se muestra en este trabajo que las diferencias de precisión entre los grupos de casos y controles son significativas -ver figuras 6 y 8- presentando la población control valores de precisión aceptables de acuerdo a trabajos de referencia previos.

Los parámetros utilizados en el índice IPI presentado en este trabajo se han utilizado en presentaciones recientes como las ya citadas de Watts et al., (2007), Siemons et al., (2009) y Hartmann, Hartmann, Heftner y Angerstein (2013) que proponen para las disfonías espasmódicas el uso del Jitter y Shimmer y la relación señal/ruido.

Vila-Rovira, Valero-García y González-Sanvisens (2011) tomaron la referencia del protocolo básico de la Sociedad Europea de Laringólogos (Dejonckere et al., 2001) el que consideraba que las medidas de perturbación de la amplitud y de la frecuencia (Shimmer y Jitter) y las de relación entre la señal y el ruido eran las más robustas para cuantificar las 
características perceptuales de la calidad vocal. No obstante, en el trabajo se señalaba que una de las limitaciones que presentaba análisis acústico informatizado era la incapacidad de analizar señales acústicas fuertemente aperiódicas. Se recomendaba no considerar los valores de perturbación de amplitud y periodo en porcentajes que superaran el 5\%. En referencia a los índices de señal / ruido se consideraba que no había suficientes estudios de estandarización. Por lo tanto Vila-Rovira et al., (2011) han identificado medidas más válidas en el estudio objetivo de la voz patológica. Refieren el uso del Dysphonia Severity Index (DSI) que investiga el Tiempo Máximo de Fonación, la Frecuencia Fundamental más aguda, la intensidad más baja y el Jitter en porcentaje. Estos autores citan la experiencia de Wuyts et al. (2000) con el DSI que ha sido seguida por varios grupos de investigadores en la búsqueda de nuevos índices, y la incorporación de medidas relacionadas con el espectro y el Cepstrum, evitando el uso del Jitter, al que Vila-Rovira y colaboradores consideran un parámetro objetivo de poca confiabilidad.

Considerando lo expuesto por los citados autores utilizamos el Jitter \% a partir de una verificación previa de la confiabilidad de la medida del F0 ciclo a ciclo (ver explicación más arriba originalidad de la propuesta). Asimismo en las emisiones de las disfonías espasmódicas se ha seleccionado segmentos de estabilidad, evitando las interrupciones o quiebres fonatorios.

Cannito et al., (2012) utilizaron el índice CPPs, una modificación del Cepstrum, como única medida acústica con valor predictivo para estimar soplosidad, con fuerte correlato acústico de aspereza. Coincidimos con Cannito en la conveniencia de usar el Cepstrum, por lo que ha sido incorporado en nuestro índice integrado IPI. De todos los indicadores presentados en la literatura, la amplitud del cepstrum es el que mejor se correlaciona con las evaluaciones perceptuales.

Como se mencionó en la introducción, los pacientes con disfonía espasmódica presentan alteraciones en el sostén de la precisión articulatoria durante la producción de la vocal por lo que resulta de interés analizar la contribución del índice IPA -ver figura 2. La modificación de los articuladores en las cavidades resonantes modifican los valores de formantes. La falta de estabilidad en la producción de los formantes es cuantificada a partir de la desviación estándar cuya inversa ha sido denominada precisión vocal articulatoria. Se muestra en este trabajo que las diferencias del índice IPA entre los grupos de casos y controles son significativas -ver figuras 6 y 8 - presentando la población control valores de precisión aceptables de acuerdo a Gurlekian y Molina (2012). 


\section{Conclusiones}

El Índice de Perturbación integrado (IPI) confirma la existencia de la disfonía estudiada con alteraciones en sus componentes acústicos fonatorios y resonanciales. El índice de Precisión Vocal Articulatorio (IPA) provee información de la inestabilidad a nivel resonancial en las voces con disfonía espasmódica. Ambos índices permiten distinguir las disfonías espasmódicas de los casos control compuesto por voces normales y con grado 1 según la escala GRBAS.

\section{Bibliografía}

Aronson, A.E, Brown, J.R., Litin, E.M., y Pearson, J.S. (1968). Spastic dysphonia II. Comparison with essential (voice) tremor and other neurologic and psychogenic dysphonia. Journal of Speech and Hearing Disorders, 33, 219-231.

Aronson A.E., y Bless, D. (1990). Clinical voice disorders. Berlin: Thieme.

Barkmeier, J., Case, J., y Ludlow, C. (2001). Identification of symptoms for spasmodic dysphonia and vocal tremor: A comparison of expert and nonexpert judges. Journal of Communication Disorders, 34, 21-37.

Braden, M.N., y Hapner, E. R. (2008). Listening: the key to diagnosing spasmodic dysphonia. ORL Head and Neck Nursing, 26, 8-12.

Brin, M.F., Blitzer, A., Stewart, C.F., Diamond, B., y Pogoda, J.M. (2001). Botulinum toxin type A for adductor spasmodic dysphonia (laryngeal dystonia): double blind placebo controlled assessment of dose and technique. Neurology, 56, 346.

Cannito, M.P., Buder, E.H., y Chorna, L.B. (2005). Spectral amplitude measures of adductor spasmodic dysphonic speech. Journal of Voice, 10, 391-410

Cannito, M.P, Buder, E., Chorna, L., y Dressler, R. (2012). Acoustic measures of phonation during connected speech in adductor spasmodic dysphonia. Laryngoscope, http://dx.doi.org/10.4172/2161-119X.S1-003

Cannito, M. P, Doiuchi, M., Murry, T., y Woodson, G.E. (2012) Perceptual structure of adductor spasmodic dysphonia and its acoustic correlates. Journal of Voice, 26, 818-823.

Cecconello, L., Farías, P., y Gurlekian, J.A. (2008). Aplicación del Cepstrum en la clínica vocal.Revista FASO, 15, 12-14. 
Dejonckere, P.H., Neumann, K.J., Moerman, M.B.J., Martens, J.P., Giordano, A., y Manfredi, C., (2012). Tridimensional assessment of adductor spasmodic dysphonia pre-and post-treatment with Botulinum Toxin. European Archives of Oto-RhinoLaryngology, 269, 1195-1203.

Edgar, J., Sapienza, C., Bidus, K., y Ludlow, C. (2001). Acoustic measures of symptoms in abductor spasmodic dysphonia. Journal of Voice, 15, 362-372.

Gurlekian, J.A. (1997). El laboratorio de Audición y Habla del LIS. En M. Guirao (Ed.), Procesos Sensoriales y Cognitivos, (pp. 55-81). Buenos Aires: Editorial Dunken.

Gurlekian, J.A., y Molina, N. (2012). Índice de perturbación, de precisión vocal y de grado deaprovechamiento de energía para la evaluación del riesgo vocal. Revista de Logopedia, Foniatría y Audiología, 32, 156-163.

Hartmann, V., Hartmann, C.J., Heftner, H., y Angerstein, W., (2013). Correlation of self assessment with expert rating and acoustic analysis for spasmodic dysphonia treatment with botulinum neurotoxin A. Basal Ganglia, 3, 165-169.

Hirano, M. (1981). Clinical examination of voice. Wien; New York: Springer-Verlag.

Kendall,K., y Leonard, R. (2011). Interarytenoid muscle botox injection for treatment of adductor spasmodic dysphonia with vocal tremor. Journal of Voice, 25, 114-119.

Ludlow, C.L., Naunton, R.F., Sedory, S.E., Schulz, G.M., y Hallett, M. (1988). Effects of botulinum toxin injections on speech in adductor spasmodic dysphonia. Neurology, $38,1220-1225$.

Pitman, M., Bliznikas, D., y Baredes, S. (2006). Spasmodic Dysphonia, 2006. http://eMedicine.com

Roy, N., Whitchurch, M., Merrill, R., Houtz, D., y Smith, M. (2008). Differential diagnosis of adductor spasmodic dysphonia and muscle tension dysphonia using phonatory break analysis, The Laryngoscope, 118, 2245-2253.

Sapienza, C.M., Walton, S., y Thomas, M. (1999). Acoustic variations in adductor spasmodic dysphonia as a function of speech task. Journal of Speech, Language and Hearing Research, 42, 127-140.

Siemons, D., Moerman, M., Marten, J.P., Deuster, D., Müller, F., y Dejonckere, P. (2009). Spasmodic dysphonia,perceptual and acoustic analysis: presenting new diagnostic tools. European Archives of Oto-Rhino-Laringology, 266, 1915-1922.

Sigal, L. (2013). Disfonías espasmódicas, estudios acústicos. Revista de Investigación en Logopedia, 2, 34-52. 
Simonyan, K., Ludlow, C., y Vortmeyer, A. (2010). Brainstem pathology in spasmodic dysphonia. The Laryngoscope, 120, 121-124.

Talkin, D. (1995). A Robust Algorithm for Pitch Tracking (RAPT). In Kleijn, W. B. and Paliwal, K. K. (Eds.), Speech Coding and Synthesis. New York: Elsevier.

Vila-Rovira J. M., Valero-García J., y González-Sanvisens, L. (2001). Indicadores fonorespiratorios de normalidad y patología en la clínica vocal. Revista de Investigación en Logopedia, 1, 35-55.

Watts, C.C.W., Whurr, R., y Nye, C. (2007). Inyecciones de toxina botulínica para el tratamiento de la disfonía espasmódica. La Biblioteca Cochrane Plus, 4.

Whitchurch, M. (2008). Adductor spasmodic disphonia versus muscle tension disphonia: Exploring the precision of phonatory break analysis as a diagnostic test. Http// www.content.lib.utah.edu/utils/getfile/.../etd2/id/.../238.pdf

Whurr, R., Lorch, M., Fontana, H., Brookes, G., Lees, y A., Marsden, C.D. (1993). The use of botulinum toxin in the treatment of adductor spasmodic dysphonia. Journal of Neurology, Neurosurgery and Psychiatry, 56, 526-30.

Wuyts, F. L., De Bodt, M., Molenberghs, G., Remacle, M., Heylen, L., Millet, B., et al. (2000). The dysphonia severity index: an objective measure of vocal quality based on a multiparameter approach. Journal of Speech, Language, and Hearing Research, 43, 796-809. 